\title{
Gravity-induced ischemia in the brain and prone positioning for COVID-19 patients breathing spontaneously: still far from the truth!
}

\author{
Mabrouk Bahloul, Sana Kharrat, Kamilia Chtara, Hedi Chelly, Chokri Ben Hamida, Mounir Bouaziz \\ Department of Intensive Care, Habib Bourguiba University Hospital and Sfax University, Sfax, Tunisia
}

\section{Dear Editor:}

In an observational study of patients admitted to the intensive care unit (ICU) [1], we showed that early application of prone positioning can improve severe hypoxemia and respiratory failure in coronavirus disease 2019 (COVID-19) patients with spontaneous breathing. In that study [1], we included 21 patients who underwent prone positioning within 6 hours following ICU admission and 17 control patients, and we concluded that early prone positioning can significantly improve severe hypoxemia. This improvement was not, however, associated with a reduction in the mortality rate or in the use of invasive mechanical ventilation $(\mathrm{P}>0.05$ for both). The results of our study are confirmed by recent larger, single- and multi-center studies and meta-analyses [2]. Moreover, to the best of our knowledge, no cases of ischemia in the brain and/or neurological complications related to prone positioning have been reported. Jaster and Ottaviani [3] suggested that the absence of improvement in the rate of mortality, or in the need for mechanical ventilation, can be fundamentally explained by "gravitational ischemia in the brain" resulting from the mass effect of one part of the brain upon another in the earth's gravitational field. These authors suggested that an upright head position (or at least partially upright) may be required to relieve gravitational ischemia in the brainstem autonomic nuclei, thereby explaining the absence of a reduction in mortality rate or in the requirement of invasive mechanical ventilation. According to this proposal, gravity behaves as an extravascular mass lesion because it forces extravascular skull or brain tissue to push against the external walls of the blood vessels, leading to brain ischemia. The authors suggest that intermittently elevating the patient's head by $30^{\circ}$ may be helpful, as it may decrease intra-cranial pressure, prevent neurological complications, and improve the outcomes of these patients. Although we agree with Jaster and Ottaviani's [3] hypothesis that gravitational consequences in the brain may contribute to delirium in the ICU in patients requiring sedation and invasive mechanical ventilation, we think that, for several reasons, this hypothesis cannot be applied in non-intubated patients with acute respiratory distress syndrome (ARDS) due to severe acute respiratory syndrome coronavirus 2 (SARS-CoV-2) infection.

First, unlike severe patients requiring sedation and mechanical ventilation, the position of patients with spontaneous breathing is usually changed from supine to prone frequently (every 2 to 4 hours), as described in most published studies [1,2], and according to Jaster and Ottaviani [3], doing so can prevent brain ischemia because it is reversible in its early stages.

\section{Letter to the Editor}

Received: February 22, 2022

Accepted: February 23, 2022

\section{Corresponding author}

Mabrouk Bahloul

Department of Intensive Care, Habib Bourguiba University Hospital, Sfax

3029, Tunisia

Tel: +216-98-69-8267

Fax: +216-74-24-3427

E-mail: bahloulmab@yahoo.fr

Copyright (C) 2022 The Korean Society of Critical Care Medicine

This is an Open Access article distributed under the terms of Creative Attributions Non-Commercial License (https:// creativecommons.org/li-censes/by-nc/4.0/) which permits unrestricted noncommercial use, distribution, and reproduction in any medium, provided the original work is properly cited. 
Moreover, as suggested by Jaster and Ottaviani [3], an upright head position (or at least partially upright), is usually used for all these patients. In fact, this position improves oxygenation in ARDS patients and was proposed as an alternative to the prone position [2].

Second, the application of the prone position leads to an increase in lung recruitability and alveolar recruitment, which causes ventilation-perfusion enhancement. As a consequence, early application of the prone position can improve severe hypoxemia and respiratory failure in COVID-19 patients with spontaneous breathing [1,2]. This improves brain oxygenation and can prevent brain ischemia. A prospectively designed, multicenter, international, randomized, open-label meta-trial with a large sample size (1,121 patients) [2] established that awake prone positioning application leads to a significant reduction in the incidence of treatment failure within 28 days of enrollment in patients with respiratory failure due to COVID-19. In this study, a longer mean daily duration of awake prone positioning ( $>8$ hours/day) was associated with treatment success at day 28 (prevention of intubation or death). For patients with acute respiratory distress requiring invasive mechanical ventilation with a partial pressure of oxygen in arterial blood/fractional percentage of inspired oxygen $\left(\mathrm{PaO}_{2} / \mathrm{FIO}_{2}\right)$ ratio $<150 \mathrm{~mm} \mathrm{Hg}$, it was established that the application of prone positioning was associated with a significant reduction of mortality [4]. According to formal guidelines [4], prone positioning is recommended for at least 16 consecutive hours in ARDS patients with $\mathrm{PaO}_{2} / \mathrm{FIO}_{2}$ ratio $<150 \mathrm{~mm} \mathrm{Hg}$ to reduce mortality (grade $1+$ ).

Third, it has been well established that the prone position improves heart function [5]. The application of the prone position leads to an increase in the cardiac preload and decreases the right ventricular afterload in patients with ARDS [5]. This results in an increase in cardiac output in patients with preload reserve, leading to positive macrocirculatory effects with enhanced organ blood flow (in particular to the brain) and oxygen delivery to the body. All these phenomena can prevent brain ischemia.

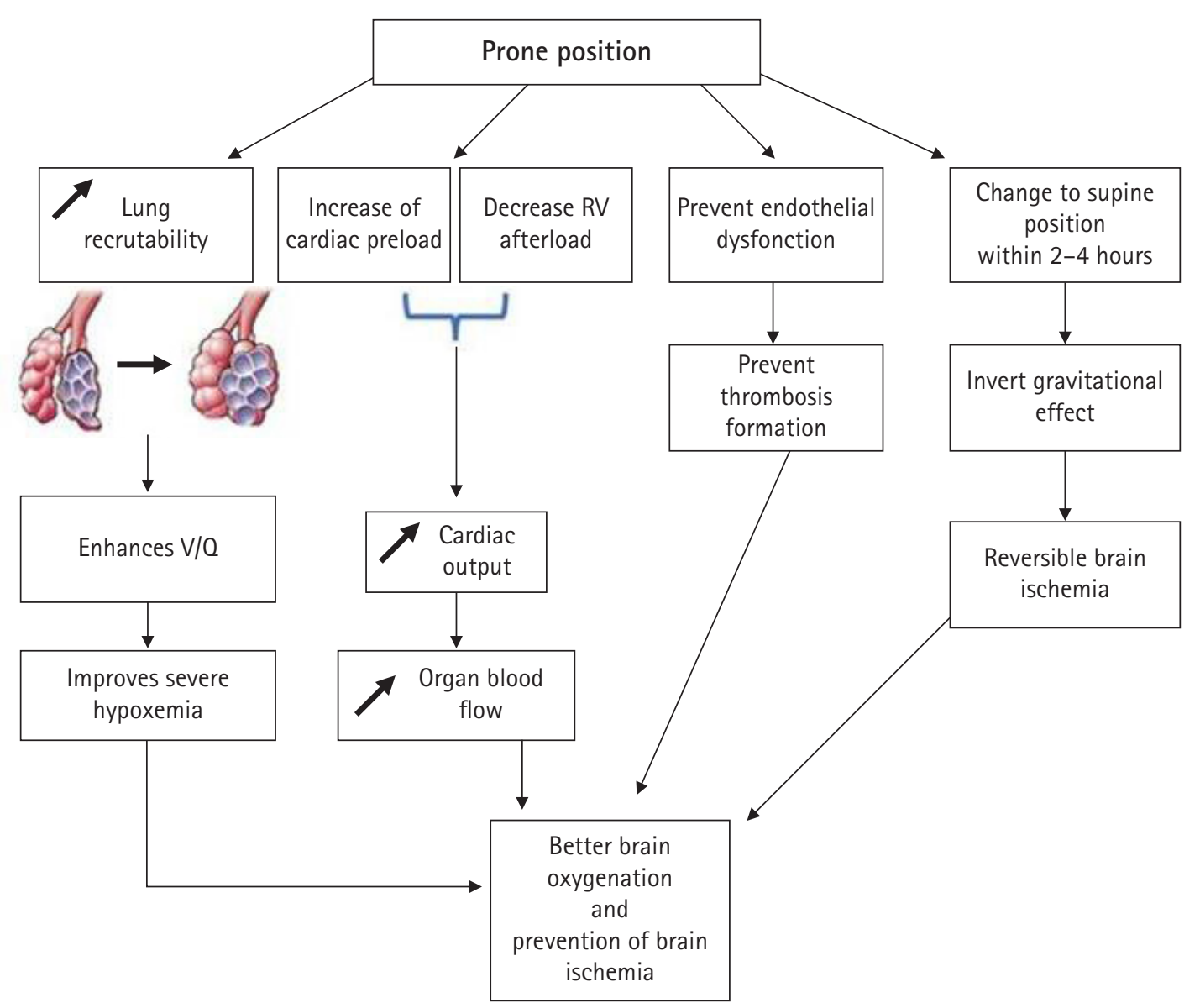

Figure 1. Impact of the prone position on brain perfusion and oxygenation. RV: right ventricular; V/Q: ventilation-perfusion. 
Finally, it has been well established that severe hypoxia leads to endothelial dysfunction and thrombosis formation. The major improvement of hypoxemia following application of the prone position can prevent this dysfunction and, as a consequence, thrombosis formation and brain ischemia.

In conclusion, as summarized in Figure 1, the early application of prone positioning can improve severe hypoxemia and outcomes in COVID-19 patients with spontaneous breathing. By substantially improving hypoxemia and cardiac output, the prone position can prevent endothelial dysfunction and, as a consequence, thrombosis formation and brain ischemia.

\section{CONFLICT OF INTEREST}

No potential conflict of interest relevant to this article was reported.

\section{ORCID}

Mabrouk Bahloul

https://orcid.org/0000-0002-6488-8294

\section{AUTHOR CONTRIBUTIONS}

Conceptualization: MB (Mabrouk Bahloul), MB (Mounir Bouaziz). Data curation: all authors. Writing-original draft: MB (Mabrouk Bahloul). Writing-review \& editing: all authors.

\section{REFERENCES}

1. Bahloul M, Kharrat S, Hafdhi M, Maalla A, Turki O, Chtara K, et al. Impact of prone position on outcomes of COVID-19 patients with spontaneous breathing. Acute Crit Care 2021;36:208-14.

2. Ehrmann S, Li J, Ibarra-Estrada M, Perez Y, Pavlov I, McNicholas B, et al. Awake prone positioning for COVID-19 acute hypoxaemic respiratory failure: a randomised, controlled, multinational, open-label meta-trial. Lancet Respir Med 2021;9:1387-95.

3. Jaster JH, Ottaviani G. Gravity-induced ischemia in the brainand prone positioning for COVID-19 patients breathing spontaneously. Acute Crit Care 2022;37:131-3.

4. Papazian L, Aubron C, Brochard L, Chiche JD, Combes A, Dreyfuss D, et al. Formal guidelines: management of acute respiratory distress syndrome. Ann Intensive Care 2019;9:69.

5. Jozwiak M, Monnet X, Teboul JL. Optimizing the circulation in the prone patient. Curr Opin Crit Care 2016;22:239-45. 